\title{
Mental illness research in the Gulf Cooperation Council: a scoping review
}

\author{
Jason E. Hickey ${ }^{1,2^{*}}$, Steven Pryjmachuk ${ }^{2}$ and Heather Waterman ${ }^{3}$
}

\begin{abstract}
Rapid growth and development in recent decades has seen mental health and mental illness emerge as priority health concerns for the Gulf Cooperation Council (Bahrain, Kuwait, Oman, Qatar, Saudi Arabia, and the United Arab Emirates). As a result, mental health services in the region are being redefined and expanded. However, there is a paucity of local research to guide ongoing service development. Local research is important because service users' experience of mental illness and mental health services are linked to their sociocultural context. In order for service development to be most effective, there is a need for increased understanding of the people who use these services. This article aims to review and synthesize mental health research from the Gulf Cooperation Council. It also seeks to identify gaps in the literature and suggest directions for future research. A scoping framework was used to conduct this review. To identify studies, database searches were undertaken, regional journals were hand-searched, and reference lists of included articles were examined. Empirical studies undertaken in the Gulf Cooperation Council that reported mental health service users' experience of mental illness were included. Framework analysis was used to synthesize results. Fifty-five studies met inclusion criteria and the following themes were identified: service preferences, illness (symptomology, perceived cause, impact), and recovery (traditional healing, family support, religion). Gaps included contradictory findings related to the supportive role of the Arabic extended family and religion, under-representation of women in study samples, and limited attention on illness management outside of the hospital setting.

From this review, it is clear that the sociocultural context in the region is linked to service users' experience of mental illness. Future research that aims to fill the identified gaps and develop and test culturally appropriate interventions will aid practice and policy development in the region.
\end{abstract}

Keywords: Arabs, Mental disorders, Mental health, Mental health services, Middle East, Qatar, Scoping review

\section{Background}

The Gulf Cooperation Council (GCC) is a union of six Arabic states in the Persian Gulf, including Bahrain, Kuwait, Oman, Qatar, Saudi Arabia and the United Arab Emirates (UAE). These countries share many cultural, political, religious, economic, and geographical similarities [1]. The aim of the union is to promote regional development through coordination, cooperation and integration among member countries [2].

The GCC has benefited substantially in recent decades from large reserves of oil and natural gas. Qatar, for example, is a small, peninsular country that was once mainly utilized by coastal fishermen, pearl divers and nomadic

\footnotetext{
* Correspondence: jehickey@ucalgary.ca

'University of Calgary Qatar, P.O. Box 23133, Doha, Qatar

University of Manchester, Oxford Rd, Manchester M13 9PL, United Kingdom

Full list of author information is available at the end of the article
}

Bedouin tribes [3]. Now, its capital city, Doha, is being transformed into an ultra-modern metropolis and the country has the highest GDP per capita in the world [4]. The UAE provides another example of rapid economic development; in less than 10 years it went from being one of the least developed countries in the world to a modern industrialized nation [5].

Islam is the foundation of cultural and social customs in the GCC [6]. It infuses nearly all aspects of life, from architecture, food choice, daily routine, social interactions, education, healthcare and more. Tolerance, hospitality and modesty are highly valued [7]. However, social customs also result in strict guidelines for what is considered appropriate behaviour in certain circumstances. For example, in Saudi Arabia, it is illegal to publically practice any religion other than Islam, and women are required to wear a gown (i.e. Abaya) and headscarf in 
public [8]. In other Gulf countries, these rules are often relaxed for foreigners. However, social and cultural norms sometimes clash with ongoing modernization and the influx of foreign workers. Because of this, some feel that traditional values are being threatened [9].

The recent and rapid changes in the region and the pressures or strains that have been mentioned above have seen mental health and mental illness emerge as priority health concerns for all countries in the GCC. In Kuwait, mental health was identified as one of six strategic priorities through a consultation with WHO [10]. As part of this agenda, mental health services are being integrated into primary healthcare, and community and home-based services are being developed. Oman also acknowledges the need to scale-up mental health services, particularly by increasing the number of available beds, providing training for primary care workers, and implementing a school health program [11]. In Qatar, a National Mental Health Strategy was recently developed [12]. This strategy focuses on system-wide change to reduce stigma, improve treatment seeking, increase availability of resources, scale-up the workforce, provide services in a variety of locations, and develop standards and guidelines. Bahrain, Saudi Arabia and the UAE have also emphasized mental health as a national priority and service development is underway in these counties as well [13-15].

Research on mental illness from GCC countries suggests that sociocultural factors influence people's experience of mental illness in the region. For example, causal attribution of mental illness to demons (Jinn) prompt people to seek traditional or religious healers frequently [16]; shame can cause families to impose social isolation on a sick family member [17]; extended family structures can promote increased levels of family support and housing [16]; and religious (Islamic) influences have been linked to non-Western presentations of illness [18]. Hence, for service planning to be most effective, it cannot necessarily rely on international best practices and evidence from other countries. However, it is widely acknowledged that there is limited local research available to guide contextually-appropriate development of mental health services in the region $[11,12]$.

The current article aims to systematically review and synthesize regional literature that reports service user perspectives on mental illness in GCC countries, identify major gaps in the literature, and suggest directions for future research. This information will facilitate the development of mental health services in the GCC. It will also provide information for mental health practitioners in non-GCC countries who provide services for Arabic people.

\section{Theoretical framework}

Arksey and O'Malley's [19] framework was used to develop the review protocol. According to one of the most commonly cited definitions, a scoping study "aims to map rapidly the key concepts underpinning a research areas and the main sources and types of evidence available..." ([20] as cited by [19], emphasis in original). Scoping studies tend to be inclusive of a range of research designs regardless of where the research sits on the 'evidence hierarchy' [21, 22] and seek to provide greater conceptual clarity [23].

Arksey and O'Malley [19] identify four possible reasons to conduct a scoping study: to examine the extent, range and nature of research activity; to determine the value of undertaking a full systematic review; to summarize and disseminate research findings; and to identify research gaps in the existing literature. Most reports on scoping studies tend to incorporate a combination of these objectives, and outcomes typically include identification of themes in the literature, gaps that have yet to be addressed, and tangible recommendations for practice and research [24-27]. These characteristics make a scoping study well-suited for the aims of the current review.

\section{Methods \\ Inclusion criteria}

Articles covering one or more of the following common or clinically relevant illnesses [28, 29] were included: mood disorders, alcohol and substance use disorders, schizophrenia, Alzheimer's and other dementias, anxiety disorders, obsessive compulsive disorder (OCD), personality disorders, and phobias. Additionally, studies had to be empirical (i.e. based on observed and measured phenomena and deriving knowledge from actual experience rather than from theory or belief), published in English, and conducted in the GCC (Qatar, Saudi Arabia, Kuwait, Bahrain, the UAE, Oman). Only studies that reported subjective data from participants were included because the perspectives and lived experiences of service users are critical for informing a recoveryoriented understanding of mental illness. Subjective data was defined as opinions or experiences collected directly from participants. Articles not meeting these criteria were excluded from the review.

\section{Identifying relevant studies}

First, several databases (CINAHL, Anthropology Plus, MEDLINE, SocINDEX, PsycINFO, Embase, and NCBI PubMed) were searched. The following search string was developed with the assistance of a librarian: ((MM "Mental Health") OR (MM "Mental Disorders +") OR (MM "Mentally Ill Persons")) AND (Cooperation Council for Arab states of the Gulf OR CCASG OR Gulf Cooperation Council OR GCC OR Qatar* OR Emirat* OR Abu Dhabi* OR Bahrain* OR Kuwait* OR Saudi Arabia* OR Oman*). Second, several regional journals (Arab Journal of Psychiatry, Eastern Mediterranean 
Health Journal, Avicenna, Journal of Local and Global Health Science, Journal of Local and Global Health Perspectives, QScience Connect, and Qatar Medical Journal) were hand searched. Databases and regional journals were searched from inception to December 2013. Finally, reference lists of articles identified in the previous two strategies were searched.

\section{Study screening}

Figure 1 illustrates the study identification and screening process. The first author screened titles from the initial database results $(n=2449)$ and removed duplicates and irrelevant articles. Inclusion criteria were then applied to full abstracts for the remaining articles $(n=655)$ by the first author. The other two authors (SP, HW) each screened a 5\% random sample of abstracts to ensure consistent application of inclusion criteria. Full papers for the articles that passed abstract screening $(n=80)$ were obtained and read in full by two authors. An additional 36 articles were excluded at this stage. Eleven articles were included from the hand search of regional journals and reference lists. Fifty-five articles were included in the final data analysis. Table 1 demonstrates how each of these 55 studies met the inclusion criteria.

\section{Data extraction}

The first author $(\mathrm{JH})$ extracted data from all 55 articles using a structured extraction template. This template was developed and piloted by the review team. Two authors (SP, HW) each extracted data from half of the final set, meaning data was independently extracted from each article twice. The two extractions were compared and discrepancies resolved through group discussions between the three authors.

Data were extracted from each study under the following categories: general information (e.g. year, profession of primary author), methodology (e.g. design, study location), sample characteristics (e.g. gender, diagnosis), results (e.g. main outcome, subjective outcome), and discussion (e.g. limitations, conclusions). Strengths and weaknesses

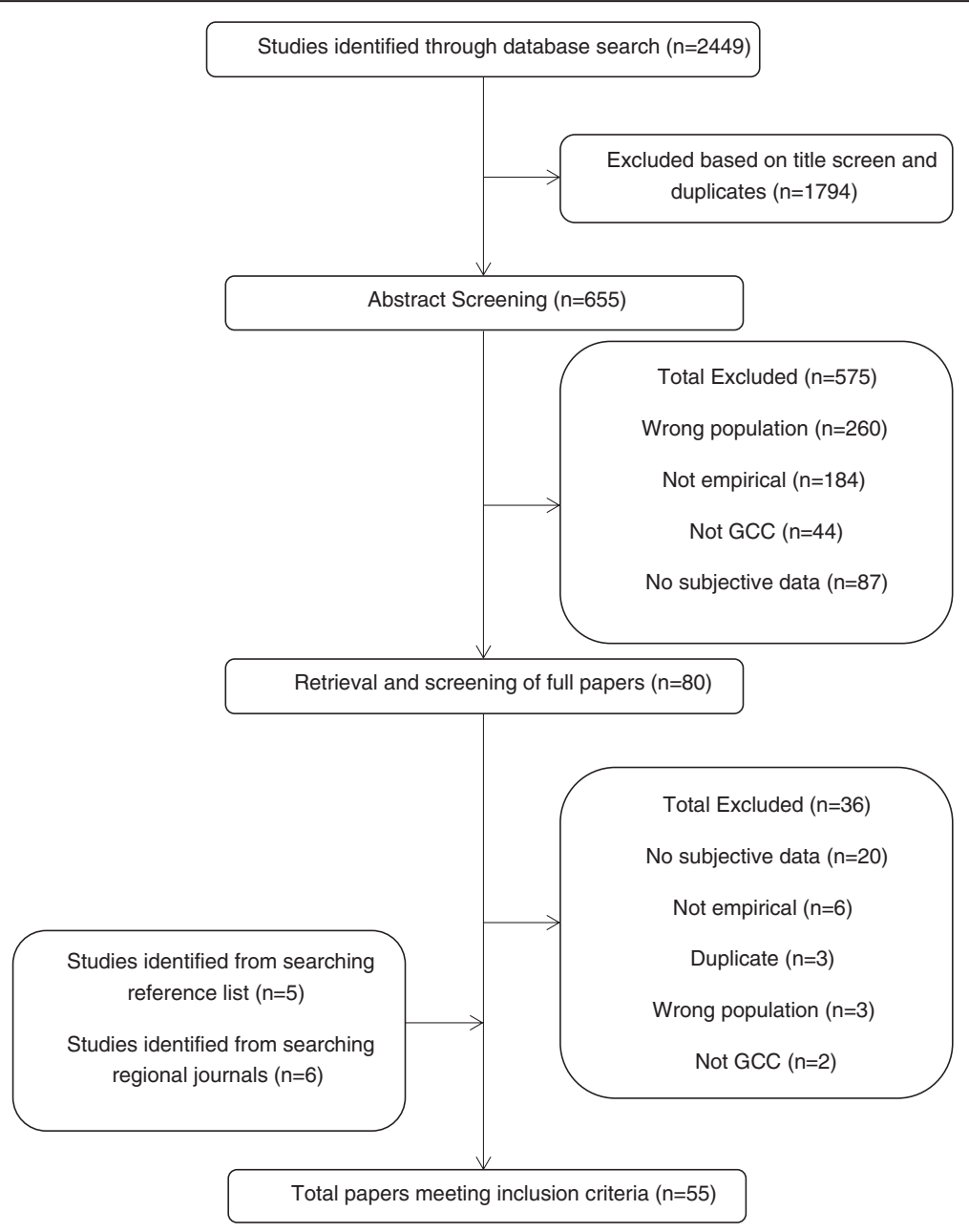

Fig. 1 Strategy used to identify studies 
Table 1 Inclusion data for all articles included in final review

\begin{tabular}{|c|c|c|c|c|}
\hline Author (date) & Design & Country & Diagnosis & Subjective data \\
\hline Al-Faraj \& Al-Ansari (2002) [58] & Cross-sectional & Bahrain & Schizophrenia & Perceived cause of symptoms/illness \\
\hline Daradkeh \& Moselhy (2011) [39] & Cross-sectional & Bahrain & Death anxiety (thanatophobia) & Degree of death anxiety and focus of anxiety \\
\hline Derbas \& al-Haddad (2001) [80] & Cross-sectional & Bahrain & Substance abuse & Factors associated with relapse \\
\hline Shooka et al. (1998) [50] & Cross-sectional & Bahrain & Obsessive compulsive disorder & Characteristics of obsessions and compulsions \\
\hline Suleiman et al. (2002) [45] & Cross-sectional & Bahrain & Depression & Symptomology \\
\hline Al-Ansari et al. (1989) [51] & Cross-sectional & Kuwait & Schizophrenia & Characteristics of hallucinations and delusions \\
\hline Al-Ansari \& Negrete (1990) [36] & Comparative cross-sectional & Kuwait & Alcohol abuse/dependence & Perception drinking patterns and associated impacts \\
\hline Al-Kandari et al. (2001) [81] & Cross-sectional & Kuwait & Substance dependence & Reasons for initiating drug use \\
\hline Al-Kandari et al. (2007) [37] & Cross-sectional & Kuwait & Substance dependence & $\begin{array}{l}\text { Preferences for illicit drugs, craving/withdrawal } \\
\text { patterns, related problems }\end{array}$ \\
\hline Al-Saffar et al. (2008) [82] & $\mathrm{RCT}$ & Kuwait & Depression & $\begin{array}{l}\text { Satisfaction with educational intervention, sources } \\
\text { of medication information }\end{array}$ \\
\hline Bilal (1988) [83] & Cross-sectional & Kuwait & Substance abuse & Perceived problems \\
\hline Bilal et al. (1987) [64] & Cross-sectional & Kuwait & Alcoholism & Reflections on illness \\
\hline Bilal et al. (1987) [64] & Cross-sectional longitudinal & Kuwait & Alcohol dependence & Problems associated with drinking \\
\hline Chaleby (1985) [57] & Cross-sectional, chart review & Kuwait & Mixed $^{\mathrm{a}}$ & $\begin{array}{l}\text { Perceptions that marriage was related to } \\
\text { psychiatric disorder }\end{array}$ \\
\hline Demerdash et al. (1981) [66] & Comparative cross-sectional & Kuwait & Substance abuse & $\begin{array}{l}\text { Reasons for drinking, reasons for combining } \\
\text { substances }\end{array}$ \\
\hline El-Islam et al. (1988) [84] & Cross-sectional & Kuwait & Depression & $\begin{array}{l}\text { Timing of onset and symptomology } \\
\text { of hypochondriasis }\end{array}$ \\
\hline El-Islam et al. (1988) [43] & Cross-sectional & Kuwait & Depression & Core depressive symptoms \\
\hline Suleiman et al. (1989) [85] & Comparative cross-sectional & Kuwait & Mixed $^{\mathrm{a}}$ & Reported negative life events \\
\hline Suleiman et al. (1986) [47] & Cross-sectional & Kuwait & Mixed $^{a}$ & Provoking factors for attempted suicide \\
\hline Zahid et al. (2010) [86] & Cross-sectional & Kuwait & Schizophrenia & Satisfaction across nine life domains \\
\hline Zahid \& Ohaeri (2010) [53] & Cross-sectional & Kuwait & Schizophrenia & Perceived caregiving burden \\
\hline Zahid \& Ohaeri (2010) [87] & Qualitative interviews & Kuwait & Schizophrenia & $\begin{array}{l}\text { Presence of hallucinations, delusions and } \\
\text { negative symptoms }\end{array}$ \\
\hline Zahid \& Ohaeri (2013) [88] & Cross-sectional & Kuwait & Schizophrenia & Met/unmet needs \\
\hline Zahid et al. (2010) [89] & Cross-sectional & Kuwait & Schizophrenia & Satisfaction with mental health services \\
\hline Zaidan et al. (2006) [42] & Cross-sectional & Oman & Alcohol abuse & Impact of alcohol abuse \\
\hline Bener et al. (2013) [90] & Cross-sectional & Qatar & Mixed $^{\mathrm{a}}$ & Reasons for non-compliance \\
\hline
\end{tabular}


Table 1 Inclusion data for all articles included in final review (Continued)

\begin{tabular}{|c|c|c|c|c|}
\hline Bener \& Ghuloum (2013) [91] & Cross-sectional & Qatar & Mixed $^{a}$ & $\begin{array}{l}\text { Satisfaction with psychiatric consultation, } \\
\text { important topics to include in consultation }\end{array}$ \\
\hline El-Islam (1982) [63] & Mixed & Qatar & Schizophrenia & Family caregiving roles \\
\hline El-Islam (1994) [48] & Cross-sectional & Qatar & Mixed $^{a}$ & $\begin{array}{l}\text { Phenomenology of various phobias, perceived } \\
\text { cause of illness }\end{array}$ \\
\hline Ghuloum et al. (2010) [92] & Prospective cross-sectional & Qatar & Mixed $^{a}$ & Satisfaction with psychiatric consultation \\
\hline Kent \& Wahass (1996) [52] & Comparative cross-sectional & Saudi Arabia & Schizophrenia & Characteristics and content of hallucinations \\
\hline Chaleby (1986) [93] & Retrospective & Saudi Arabia & Alcohol/substance abuse & Frequency of stressors \\
\hline Chaleby \& Raslan (1990) [94] & Cross-sectional & Saudi Arabia & Social phobia & $\begin{array}{l}\text { Perceptions of childhood, parents, work } \\
\text { environment }\end{array}$ \\
\hline Mahgoub \& Abdel-Hafeiz (1991) [49] & Cross-sectional & Saudi Arabia & Obsessive compulsive disorder & Characteristics of obsessions and compulsions \\
\hline Shahin \& Daly (1999) [95] & Cross-sectional & Saudi Arabia & Mixed $^{a}$ & $\begin{array}{l}\text { Knowledge, attitudes and beliefs about } \\
\text { psychiatric medications }\end{array}$ \\
\hline Abalkhail (2001) [35] & $\begin{array}{l}\text { Prospective, } \\
\text { comparison group }\end{array}$ & Saudi Arabia & Substance dependence & Symptoms experienced during detox \\
\hline Al Sughayir (2000) [96] & Case-control & Saudi Arabia & Mixed $^{a}$ & Possession beliefs \\
\hline Al-Habeeb et al. (2013) [43] & Cross-sectional & Saudi Arabia & Depression & $\begin{array}{l}\text { Components of suicidal ideation, frequency/duration } \\
\text { of suicidal ideation, control over suicidal thoughts, } \\
\text { reasons and deterrents } \\
\text { for attempting suicide }\end{array}$ \\
\hline Al-Habeeb \& Qureshi (2000) [56] & Cross-sectional & Saudi Arabia & Mixed $^{a}$ & $\begin{array}{l}\text { Reasons for smoking, reasons for not smoking, } \\
\text { associated impacts, experiences with } \\
\text { smoking cessation }\end{array}$ \\
\hline Al-Nahedh (1999) [65] & Cross-sectional & Saudi Arabia & Substance abuse & Reasons for initiating drug use \\
\hline Alshowkan et al. (2013) [97] & Cross-sectional & Saudi Arabia & Schizophrenia & Perceived quality of life \\
\hline Al-Solaim \& Loewenthal (2011) [34] & Qualitative interviews & Saudi Arabia & Obsessive compulsive disorder & $\begin{array}{l}\text { Encounters with traditional healers, supernatural } \\
\text { beliefs, impact of religion, impact of illness }\end{array}$ \\
\hline Al-Subaie (1994) [61] & Cross-sectional & Saudi Arabia & Mixed $^{a}$ & Perceived outcome of traditional healing \\
\hline Chaleby (1988) [98] & Cross-sectional & Saudi Arabia & Mixed $^{a}$ & $\begin{array}{l}\text { Factors perceived to be associated with } \\
\text { marital discord }\end{array}$ \\
\hline Daradkeh \& Al Habeeb (2005) [99] & Cross-sectional & Saudi Arabia & Schizophrenia & Perceived level of health and quality of life \\
\hline lqbal (2002) [40] & Cross-sectional & Saudi Arabia & Substance abuse & $\begin{array}{l}\text { Characteristics of hearing changes after } \\
\text { amphetamine use }\end{array}$ \\
\hline Qureshi (1992) [41] & Cross-sectional & Saudi Arabia & Substance abuse & $\begin{array}{l}\text { Reasons for using, symptoms associated with } \\
\text { craving, methods to obtain drug }\end{array}$ \\
\hline Qureshi et al. (1998) [62] & Cross-sectional & Saudi Arabia & Mixed $^{a}$ & $\begin{array}{l}\text { Reasons for seeking, and perceived outcomes } \\
\text { of, traditional healing }\end{array}$ \\
\hline
\end{tabular}


Table 1 Inclusion data for all articles included in final review (Continued)

\begin{tabular}{|c|c|c|c|c|}
\hline Zarrouk (1975) [54] & Cross-sectional & Saudi Arabia & Schizophrenia & Characteristics of hallucinations \\
\hline Zarrouk (1978) [55] & Cross-sectional & Saudi Arabia & Schizophrenia & Characteristics of hallucinations and delusions \\
\hline $\begin{array}{l}\text { Al Zarrad \& Abu-Mugaiseeb } \\
\text { (2002) [100] }\end{array}$ & Prospective & UAE & Mixed $^{a}$ & Attitudes and satisfaction towards service \\
\hline Amin \& Hamdi (1995) [32] & $\begin{array}{l}\text { Retrospective chart review, prospective } \\
\text { follow-up }\end{array}$ & UAE & Mixed $^{a}$ & Symptomology \\
\hline Daradkeh \& Karim (1994) [60] & Cross-sectional & UAE & Schizophrenia & Negative impacts of illness \\
\hline Hamdi et al. (1997) [44] & Cross-sectional & UAE & Depression & Characteristics of depressive symptomology \\
\hline Salem et al. (2009) [33] & Cross-sectional & UAE & Mixed $^{a}$ & $\begin{array}{l}\text { Outcomes after consultation with traditional healer, } \\
\text { perceived cause of illness }\end{array}$ \\
\hline
\end{tabular}


of individual studies were assessed and recorded during the extraction process.

\section{Data analysis}

Descriptive statistics were compiled to illustrate the "extent, nature and distribution" [19] of the literature identified. A thematic analysis of the subjective data was also conducted. The purpose of this analysis was to identify and elaborate on the main concepts addressed by the literature. One of the main criticisms of scoping studies is that there is a lack of transparency and rigour in synthesizing and presenting thematic results. Framework analysis [30] was chosen as an analytic approach in order to address these issues.

Framework analysis proceeds through a series of logical steps to reach a narrative summary of the results. Data extraction forms were read and re-reread to increase familiarity, then codes were applied to identify key concepts within the data. Once all extraction forms had been coded, the initial codes were reviewed and revised to create a conceptual framework. The conceptual framework was then applied back to the extraction form to assess its fit. When the extraction forms had been recoded according to the conceptual framework, the data was entered into a matrix where results could be examined across themes (columns) or article (rows). The majority of analysis was conducted by one author $(\mathrm{JH})$. However, initial codes and a draft of the conceptual framework was reviewed and discussed by the entire team, and the matrix was reviewed to ensure logic and consistency.

\section{Results}

Figure 2 displays the publication timeline. The oldest included study was published in 1975 and there are three notable peaks in publication frequency in 1988, 2001-2002, and 2010-2013. There is an upward trend in publications over the entire period.
The greatest number of studies were conducted in Saudi Arabia $(n=21,38 \%)$ followed by Kuwait $(n=18,33 \%)$. Five studies (9\%) were conducted in each of Qatar, Bahrain and the UAE. One study (2\%) was conducted in Oman.

Psychiatrists acted as first author on the majority of publications $(n=39,70 \%)$ followed by researchers from medicine (specialty unspecified) and nursing, who authored four papers each (7\%). Professions of other first authors included epidemiology $(n=2,4 \%)$, psychology $(n=2,4 \%)$, the behavioural sciences $(n=1,2 \%)$, and pharmacy $(n=1,2 \%)$. The profession of the primary author was unspecified in two cases.

Figure 3 displays which diagnoses were investigated. The most common target was a mixed sample $(n=16,28 \%)$, comprising individuals with a range of diagnoses. This was followed by studies examining exclusively schizophrenia $(n=14,25 \%)$ and alcohol or substance use disorders $(n=13,23 \%)$. None of the included articles targeted Alzheimer's or other dementias.

Table 2 displays sample size by gender and diagnosis. Overall, males outnumber females by a ratio of 2.2 to 1 . The largest gender imbalance occurred for alcohol or substance use disorders where males outnumbered females by a ratio of 72.6 to 1 . The only disorder where females were over represented was OCD, where females outnumbered males by a ratio of 2.6 to 1 .

The vast majority of studies $(n=42,84 \%)$ recruited participants from public psychiatric treatment centres. Of these, half recruited from inpatient or detox units, while half recruited from outpatient departments. Two studies (4\%) recruited from private clinics, and only one study (2\%) recruited participants from the community. Twelve studies $(24 \%)$ were unclear about recruitment location.

Several themes and sub-themes emerged during analysis, including service preferences, illness (perceived cause, symptomology, impact), and recovery (traditional healing, family support, religion). The following section, which

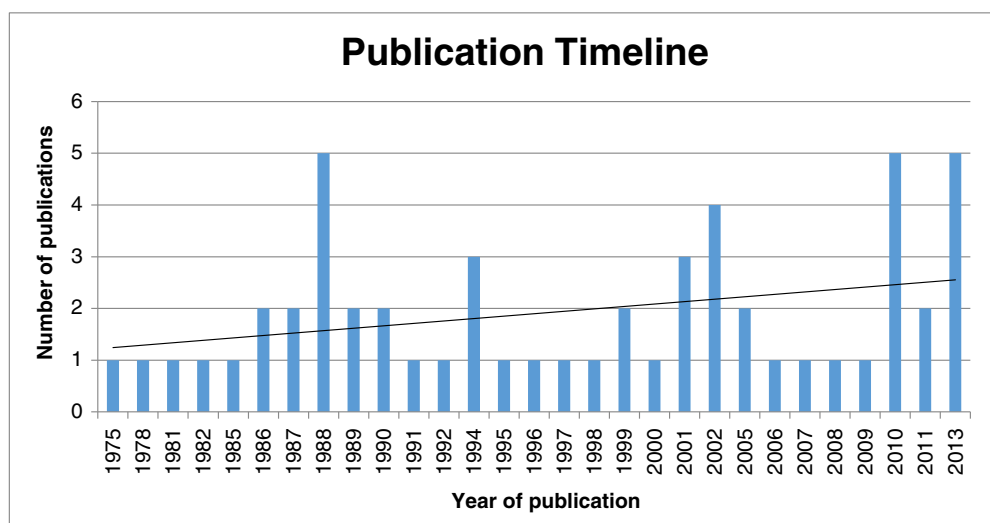

Fig. 2 Number of publications by year of publication 


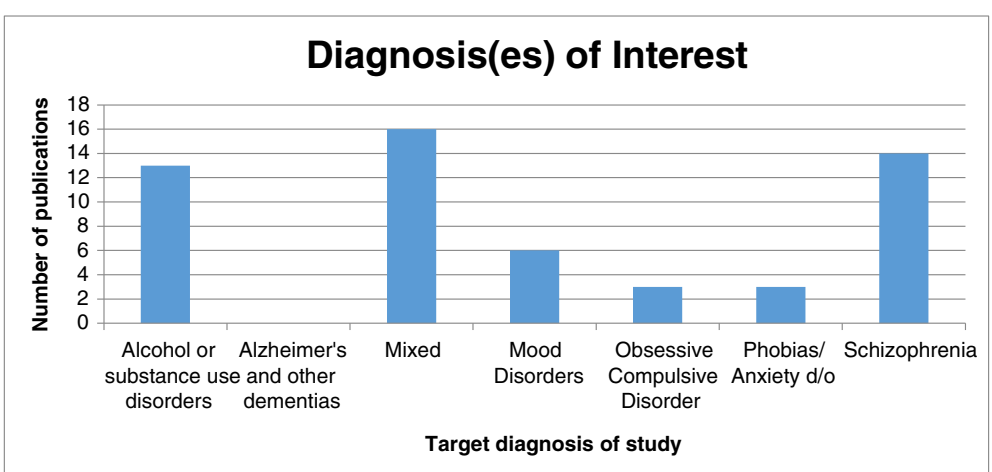

Fig. 3 Number of publications by target diagnosis

critically synthesizes the results of this review, is organized according to these themes and sub-themes.

\section{Service preferences}

Gender seemed to influence service preferences in several studies. For example, Bener and Ghuloum [31] found that patient gender affected the type of topics seen as most important when receiving treatment from a psychiatrist. In that study, males viewed discussions with a doctor about treatment options as being most important, while females prioritized explanations of the condition and the underlying cause. Surveyed preferences were limited to interactions with a psychiatrist; thus, it is not possible to determine preferences across other professional groups. Additionally, the results were collected using a brief questionnaire, which limited choices and did not explore the underlying reasons for observed differences. However, the study suggests that gender is an important consideration for service delivery in this region.

The importance of gender is reinforced by Amin and Hamdi [32], who found gender to have an influence on where participants preferred to seek treatment. The authors found that females in need of psychiatric care tend to present at the emergency department, while males were more often seen in the outpatient department. Amin and Hamdi [32] suggest several reasons for their observed

Table 2 Gender of participants for each of the studied diagnostic categories

\begin{tabular}{llll}
\hline Diagnosis of interest & Male & Female & Ratio \\
\hline Alcohol or substance use disorders & 1452 & 20 & 72.6 to 1 \\
Depression & 668 & 263 & 2.5 to 1 \\
Obsessive compulsive disorder & 27 & 70 & 1 to 2.6 \\
Phobias/anxiety disorder & 165 & 65 & 2.5 to 1 \\
Schizophrenia & 1111 & 413 & 2.7 to 1 \\
Mixed sample & 2704 & 1958 & 1.4 to 1 \\
Total & 6127 & 2789 & 2.2 to 1 \\
\hline
\end{tabular}

difference but these were conjecture and not grounded in the results. Additionally, this study is now 20 years old and with the recent modernization in the region, results may no longer be accurate. Thus, there has been no recent research to examine service users' preferences across a range of services within the healthcare system.

Other studies, however, have demonstrated a preference for services outside of the healthcare system. Salem et al. [33] found that nearly half of the sample went to a faith healer prior to seeking psychiatric care. The majority of these participants continued to see a faith healer even after engaging with psychiatric services. Because convenience sampling was used in this study it is difficult to generalize results to the wider population. However, the participants originated from various countries in the Gulf region, had a range of diagnoses, and included nearly equal proportions of men and women. This diverse sample adds to the generalizability of the study. The author concludes that mental health professionals need to be aware of patient preferences for traditional healing and understand the reasons why they sometimes refuse medical treatment.

A comparable study conducted by Al-Solaim and Loewenthal [34] in Saudi Arabia demonstrated that psychiatric services seem to be seen as a last resort when other options (e.g. faith healers) are not successful. This reinforces Salem et al.'s [33] argument that mental health professionals should not ignore the contribution of traditional healers to service users' treatment. However, Al-Solaim and Loewenthal's [34] results are drawn solely from the experiences of 15 women. It is possible that they may not represent the majority view. Men, in particular, as demonstrated earlier, may have different preferences.

\section{Illness: symptomology}

Symptomology was one of the most commonly occurring themes in the included articles. Clinical presentation of mental illness was described for alcohol and 
substance abuse [35-42], depression [32, 43-45], suicide $[46,47]$, panic disorder [48], OCD [34, 49, 50], schizophrenia [51-55], smoking behaviour [56], and hypochondriasis [18]. Several of these articles emphasized the unique presentation of certain illness within the Arab context.

For example, Shooka et al. [50] demonstrated that religious and blasphemous thoughts were the most common obsessions in patients with OCD, while repeated prayer-related cleaning and washing was the most common compulsion. The authors suggest that there are higher levels of religious content in participants from a strict religious context. These findings were reinforced by Mahgoub and Abdel-Hafeiz [49], who found an Islamic focus for obsessions and compulsions in OCD. In particular, prayer and body washing were the most common obsessions and religious repeating and religious washing were the most common compulsions. Both these studies are quite old; however, the Islamic influence on symptomology was also apparent in Al-Solaim and Loewenthal's [34] more recent study on OCD. An additional finding in this more recent study was that symptoms in the religious domain were the most disturbing for patients and their families. Despite their limitations, these studies suggest that the content and focus of OCD symptoms are culturally influenced.

Research on other disorders has also demonstrated a contextual influence. For example, a study by Kent and Wahass [52] on schizophrenia in Saudi Arabia found that hallucinations had more religious and superstitious content compared to a sample from the United Kingdom. Zarrouk [55] found that delusions also differed from nonArabic samples, with Saudi patients more frequently believing they were being 'made' to do things. In a study on depression by Hamdi et al. [44], four main types of symptom variation (compared to non-Arabic studies) were identified, namely variations in idioms (e.g. heavy/tense vs. depressed/sad), use of somatic metaphors (e.g. 'my body is shattered'), influence of religion (e.g. denying acts considered haraam/forbidden), and behavioural alterations (e.g. going into desert to stare at nothing). Several of these variations clearly reflect the local context.

Taken together, these articles demonstrate a contextual influence on symptomology in the GCC. It should be noted that religion is also associated with symptoms in non-Arabic contexts. However, the religious content of symptoms in this review are clearly shaped by the Islamic context. This finding has appeared repeatedly over a considerable period of time (1978-2011), which suggests that this is a somewhat stable phenomenon. Many of these authors reasonably suggest that an understanding of local variations in clinical presentation is important for accurate diagnosis and treatment of service users.

\section{Illness: perceived cause}

The perceived cause of mental illness was often external. For example, participants with a diagnosis of nosophobia attributed the cause to over-investigation of minor complaints by physicians [48]. Similarly, over one-third of women in polygamous marriages attributed their illness to their marriage [57], the majority of caregivers attributed the cause of illness to social stressors [58], and those with thoughts of self-harm attributed these thoughts to the devil [48]. Taken individually, the design weaknesses (e.g. convenience sample, bias, cross-sectional design) in these articles prevent reliable conclusions from being made. However, the consistent external causal attribution across studies lends credibility to the findings.

One particular class of perceived external cause, the supernatural (e.g. black magic, jinn, evil eye), seems particularly common. For example, Salem et al. [33] found that about one-third of participants attributed their illness to supernatural factors, while one-third attributed them to psychiatric problems and one-third were unsure. Similarly, Al-Solaim and Loewenthal [34] and Al-Sughayir [59] found that the majority of patients attributed their illness to possession by jinn (i.e. evil spirits). This external attribution to supernatural forces can be protective as certain symptoms may not be as stigmatized and may not produce such strong feelings of guilt $[34,48]$. In fact, those with the evil eye may see themselves as having some positive attribute worthy of envy, which boosts their self-esteem [34]. Again, there are design weaknesses (e.g. bias, unrepresentative samples), but the consistent findings on supernatural attribution warrant further, more rigorous investigation.

\section{Illness: perceived impact}

Most of the data under this theme come from studies on alcohol and substance abuse, which limits applicability to people with other diagnoses. Additionally, there was a certain amount of heterogeneity in impact outcomes. For example, Al-Ansari and Negrete [36] surveyed people undergoing treatment for alcohol abuse. Participants felt guilty about drinking, that it caused their family/friends to worry about them, that it sometimes created interpersonal problems, and caused them to neglect personal responsibilities. In contrast, Zaidan et al. [42] found that the majority of their sample never felt guilty about their drinking. Based on the information presented, the samples in the two studies seem comparable, aside from country of residence. Hence, the reasons for the observed differences are unexplained. Additionally, both studies enrolled males only and therefore results are further limited.

Daradkeh and Karim [60] and Al-Solaim and Loewenthal [34] offer limited information on illness impact among people with schizophrenia and $O C D$, respectively. These included barriers to social inclusion (schizophrenia) and 
interpersonal problems with family members (schizophrenia and OCD). However, the small sample size used in these studies means that further investigation is needed before reliable conclusions can be made.

Another major limitation in the majority of the studies on alcohol and substance abuse is their almost exclusive reliance on questionnaires to collect data. This limits the depth and richness of information collected and prevents participants from sharing relevant information that is not covered on the questionnaire. Thus, despite the relatively large number of studies on this topic, understanding remains limited.

\section{Recovery: traditional healing}

As mentioned above, a large proportion of psychiatric patients visit a traditional healer prior to seeking medical help [32-34, 58, 61]. This practice was perceived to contribute to recovery in a variety of ways. However, there were mixed perceptions on the effectiveness of traditional treatment.

Qureshi et al. [62] found that some participants with depressive or catatonic symptoms reported a temporary improvement from traditional treatment but that most were unsatisfied. Similarly, Salem et al. [33] found that about half of participants with a range of diagnoses experienced only a temporary benefit, with others experiencing no benefit at all. Both studies interpreted 'benefit' as a reduction in symptoms.

While most authors exploring the effectiveness of traditional healing seem to assume that symptom reduction is of primary importance, this may not necessarily be the case for service users. For example, Al-Subaie [61] found that, even those who did not perceive their symptoms to be reduced, reported feeling that God would reward them for having faith in traditional healing methods, which are primarily based on religious beliefs. This finding suggests that service users may place value on treatment benefits other than a reduction of symptoms. However, these other potential benefits are largely ignored in the studies reviewed, as is the relative importance of various benefits to participants.

A further limitation in the studies included in this section is selection bias. Since participants were selected from the population of people seeking psychiatric treatment, it is possible that others received longer lasting benefit from traditional treatments and did not subsequently access services. A broader sampling strategy (e.g. including people with mental illness who do not make regular use of psychiatric services) would be necessary for a more accurate investigation.

\section{Recovery: family support}

The extended nature of the Gulf Arab family was frequently addressed as being a source of support for service users. Potential supportive roles of the family in one study included medication supervision, being tolerant of short periods of withdrawal, helping to find acceptable ways to describe and understand the illness, not expecting anything in return for their help, and assistance in filling leisure time [63]. While these caregiving themes make sense intuitively, the author introduces subjectivity and bias into the analysis and does not consider alternate or contradictory views. Additionally, the study was conducted over 20 years ago and no similar studies have been conducted to support or refute the author's findings. It is also possible that the supportive role of families has changed in the ensuing period of rapid socioeconomic development.

Studies also suggested that support is more common in extended families compared to nuclear families and that those living in extended families had more social contact, better personal hygiene, less active symptoms, and better treatment outcomes [63, 64]. Conversely, the extended family was also perceived to have a negative impact; tension or stress within the family were cited as reasons for substance abuse [65] and attempted suicide [47], and were associated with higher disease severity [53]. Unfortunately, only the study by Zahid and Ohaeri [53] was recent enough to consider these influences within a modern context, and even this study did not examine the issue in depth. Hence, while it seems likely that the family plays a role in service users' recovery from mental illness, the nature of this role in a modern context remains unclear.

\section{Recovery: religion}

Committing acts that were incongruent with the teachings of Islam led to feelings of guilt and lower self-esteem $[34,48]$. The more compliant a person was with the values of their faith, the more pride they felt [34]. However, compulsive religious acts relating to mental illness sometimes interfered with daily life and led to treatment seeking. Religion, however, particularly prayer, was still seen as one of the main ways to cope with mental illness and related stress. For example, being religious was associated with lower levels of death anxiety [39] and alcohol abuse [64, 66].

The majority of the studies included in this section incorporated religion as a minor variable that was a small part of a larger study. Thus, a systematic, in-depth investigation of religion has not been undertaken. Despite a lack of direct evidence, many of the reviewed studies argued that religion had an impact on certain aspects of participants' illness, including symptomology (e.g. [43]), treatment seeking (e.g. [37]), etc. However, these claims and their underlying assumptions are currently unsubstantiated by the literature. In other words, broad assumptions were sometimes made, based solely on the 
authors' own personal beliefs or professional understanding about the value of religion. Thus, while it seems likely that religion plays a role, it is difficult to objectively interpret the impact of religion based on the literature included in this review.

\section{Discussion}

This systematic scoping review was undertaken in order to synthesize regional literature on service users' experience of mental illness, identify gaps in the literature, and identify opportunities for future research; 55 articles were included in the review.

The included studies offer a small glimpse into service preferences, including a preference for initial consultations with faith healers. The use of faith healers to treat mental illness has also been documented in other developing countries. For example, a study conducted in rural India reported that faith healing is widely used and that many people seek traditional services before medical psychiatric services [67]. Services of faith healers are also commonly used in Ghana. This is because traditional healers offer more culturally appropriate models of understanding illness, higher levels of psychosocial support, and easier accessibility [68]. A study in Zimbabwe found that three quarters of people sought both traditional and medical treatment for mental illness; however, in this study, biomedical care providers were the most common point of first contact [69].

The frequent use of traditional healers has implications for government-run psychiatric services. The title of the article by Ae-Ngibise et al. [68] summarizes these implications quite well: 'Whether you like it or not, people with mental health problems are going to go to them [faith healers]'. While some collaboration currently occurs in Qatar, ongoing service development efforts should aim to identify opportunities to incorporate safe, appropriate traditional healing as part of a comprehensive service.

External stressors, particularly the supernatural (i.e. jinn, black magic, evil eye) were frequently seen as the root cause of mental illness. This indicates a need to address cultural beliefs and social factors when treating mental illness. A recently published review found that psychiatric symptoms are commonly attributed to the supernatural among Muslims worldwide [70]. The authors claim that this external attribution has diagnostic and treatment implications. For example, biomedical treatment may not be accepted if underlying cultural beliefs about the supernatural are not addressed [71]. Therefore, practitioners should foster an awareness of traditional beliefs and be open to incorporating these as part of the therapeutic process.

Symptomology was described for most major mental illnesses and several similarities and differences were identified compared to typical presentations in the West. Variations in presentation of mental illness across cultures has been widely reported and debated in the literature [72]. Variations create difficulties in applying standard diagnostic criteria such as the DSM- 5 or the ICD-10 in cultural contexts that differ from the West. This provides fuel for an argument that the dichotomous nature of diagnostic categories are unhelpful in the treatment of people with mental health issues [73, 74]. Unlike physical illness, mental illness is predominantly subjective (i.e. unmeasurable); interpretation and explanation of symptoms by the patient is influenced by sociocultural understanding [75, 76]. Attending to a person's subjective experience of their illness and the overall impact of the illness on their life can lead to a more holistic understanding of the patient and better treatment outcomes [77]. It is worth noting that this practice aligns well with recovery-oriented care.

Other sociodemographic and sociocultural factors, such as family support, marriage, religion, education, financial status, gender, birth order, and nationality/ethnicity, were also investigated. However, the relationship of these factors to mental illness was difficult to assess due to limited data and weaknesses of the included studies. These areas provide fertile ground for future research.

The articles included in this review add important, contextually-relevant data to our understanding of mental illness in the region. However, despite nearly 40 years of research addressing the impact of sociocultural factors on people's experiences of mental illness, a limited understanding of these issues remain. For example, the Arab extended family is widely acknowledged in having a key supportive role for those with mental illness. However, it has also been reported as a source of stress and conflict. Religion influences all aspects of life in Arab society and provides a source of strength and support for people with mental illness. However, forbidden acts, such as alcohol and substance abuse and suicide, still occur, and cause guilt and worry for patients and their families. Widespread belief in the influence of the supernatural on mental illness has been documented. These beliefs fall within the scope of modern mental health practice, but there is little evidence of how they can be incorporated successfully into a contextually-relevant model of psychiatric care and recovery. Research into the interplay between psychiatry and traditional healing is also lacking; service users place value on consultation with faith healers, but it is unclear how these services might be incorporated into a cohesive system of mental health service in the region, or even if they should be incorporated. The concept of stigma is conspicuously absent from the articles and little effort has gone into measuring or describing stigma towards mental illness in the GCC. Also absent from the literature are investigations into service users' self-management of their 
illness. Most service users spend the majority of their lives outside of the healthcare system. Yet, virtually nothing is known about the strategies and resources they use to minimize the day-to-day challenges of mental illness.

\section{Strengths and limitations}

This review is the first of its kind to be conducted in the Gulf region. Two other reviews of mental health research have been published [78, 79]. However, the scope of these reviews is limited to the frequency, distribution and topics of publications. This review is the first to synthesize the results of research on mental illness in this region. Additionally, the systematic approach undertaken for this review facilitated a relatively objective synthesis that was rigorously conducted. Finally, the use of Framework Analysis overcomes a major criticism of scoping studies by providing a transparent method of analysis where thematic results can be clearly linked to individual studies.

This review is limited by the general weaknesses in the body of literature. For example, most of the research included in this review seems to be unfunded. This implies that proposals may not have been subjected to peer review. While lack of funding does not necessarily imply low quality, many of the papers reviewed here would have benefitted from additional peer review during the planning stage. Increasing the funding available for mental health research could help to improve the overall quality of the research (e.g. through the scientific review process) and guide the focus of future research to ensure it is ethically sound and relevant for the development of practice and policy.

The evidence base consists primarily of cross-sectional studies aimed at developing foundational knowledge. This type of evidence does not allow for causal inferences to be made and only provides a snapshot of a phenomenon at one point in time. This means that the relationships between sociodemographic factors and illness experiences are still not well understood. Longitudinal research would help to increase understanding of illness trajectory outside of the hospital setting, while interventional research would help to improve the transformation of knowledge into practice. Additionally, the vast majority of included research was quantitative, meaning that results of the review lack depth. Incorporating more qualitative research has the potential to clarify important issues, help to develop a better understanding of service users' perspectives, and build contextually relevant mental health theory that can be applied and tested through subsequent research. Qualitative research would also facilitate development of contextually valid measurement scales and questionnaires.

Underrepresentation of women in the studies highlights the need to look beyond convenience samples in psychiatric research at ways of identifying more representative samples. The gender imbalance implies that review findings are more relevant to the male psychiatric population. Finally, very few studies include family members or caregivers, even though most acknowledge the contribution of social support to recovery. Thus, understanding of caregivers' potentially supportive role in the region remains limited.

\section{Conclusion}

Despite the limitations of the reviewed literature, we can conclude that the sociocultural context in the Gulf region is linked to people's experience of mental illness. However, service users' perceptions and understandings about the nature of the context-illness experience relationship have not been systematically explored. This is particularly true for the process of self-management of illness outside the hospital setting. Interventions that are developed based on this limited understanding may have limited effectiveness and acceptability. While many questions can be derived from the identified knowledge gaps, two seem prominent:

1. What strategies do service users in Arabic countries use to self-manage their mental illness outside of the hospital setting?

2. What treatments and interventions would be most effective and acceptable to support this self-management?

Future research that attempts to address these two questions will increase the capacity of Arabic mental health services to provide efficient and culturally appropriate support to service users.

\section{Authors' contributions \\ All authors made substantial contributions to the conception and design of this study. JH was responsible for article retrieval, study screening, data extraction and analysis, and manuscript preparation. SP and HW participated in study screening, data extraction and analysis, and critical review and revision of the manuscript. All authors read and reviewed the final manuscript.}

\section{Authors' information}

JH has been working as a Nursing Instructor in Qatar since 2012. His research focuses on service users' self-management of mental illness in the Middle East. This article was undertaken as a part of JH's PhD studies at the University of Manchester.

\section{Competing interests}

The authors declare that they have no competing interests.

\section{Author details}

${ }^{1}$ University of Calgary Qatar, P.O. Box 23133, Doha, Qatar. ${ }^{2}$ University of Manchester, Oxford Rd, Manchester M13 9PL, United Kingdom. ${ }^{3}$ Cardiff University, Cardiff CF10 3XQ, United Kingdom.

Received: 22 November 2015 Accepted: 24 June 2016

Published online: 04 August 2016 


\section{References}

1. AIMarri TSK, Oei TPS. Alcohol and substance use in the Arabian Gulf region: A review. Int J Psychol. 2009;44:222-33.

2. Cooperation Council for the Arab States of the Gulf. Cooperation Council for the Arab States of the Gulf. The Charter. Cooperation Council for the Arab States of the Gulf; 2012. http://www.gcc-sg.org/en-us/AboutGCC/ Pages/Primarylaw.aspx. Accessed 27 Mar 2016

3. Kronfol Z, Ghuloum S, Weber A. Country in focus: Qatar. Asian J Psychiatr. 2013;6:275-7.

4. Knoema. GDP per Capita Ranking 2015. 2015. http://knoema.com/sijweyg/ gdp-per-capita-ranking-2015-data-and-charts. Accessed 9 June 2016.

5. Shihab M. Economic Development in the UAE. In: Abed I, Hellyer P, editors. United Arab Emirates: a new perspective. London: Trident Press Ltd.; 2001. p. 249-59.

6. Williams J. Don't They Know It's Friday? Cross-Cultural Considerations for Business and Life in the Gulf. 1st ed. London: Motivate Publishing; 1999.

7. Government of Dubai Executive Council. The Dubai Code of Conduct. http:// www.zu.ac.ae/employment/html/documents/CultureandConductinDubai_000. pdf. Accessed 9 Jun 2016

8. Arabia S. Local laws and customs. 2016. https://www.gov.uk/foreign-traveladvice/saudi-arabia/local-laws-and-customs. Accessed 10 April 2016.

9. Fellow J. Qatar's delicate balancing act. BBC News. 2013. http://www.bbc.co. uk/news/world-middle-east-21029018. Accessed 9 Jun 2016.

10. World Health Organization. Country Cooperation Strategy for WHO and Kuwait 2012-2016. Cairo: WHO Regional Office for the Eastern Mediterranean; 2014

11. World Health Organization. Country Cooperation Strategy for WHO and Oman 2010-2015. Cairo: WHO Regional Office for the Eastern Mediterranean; 2010.

12. Supreme Council of Health. Qatar National Mental Health Strategy. Changing Minds, Changing Lives: 2013-2018. 2013. http://www.nhsq.info/ app/media/1166. Accessed 15 December 2013.

13. Qureshi NA, Al-Habeeb AA, Koenig HG. Mental health system in Saudi Arabia: an overview. Neuropsychiatr Dis Treat. 2013;9:1121-35.

14. Kingdom of Bahrain Ministry of Health. Bahrain Health Strategy. Framework for Action. Bahrain: Ministry of Health; 2002.

15. World Health Organization. Country Cooperation Strategy for WHO and United Arab Emirates 2012-2017. Cairo: WHO Regional Office for the Eastern Mediterranean; 2012

16. El-Islam M. Arab culture and mental health care. Transcultural Psychiatry. 2008:45:671-82.

17. Al-Krenawi A, Graham JR. Culturally sensitive social work practice with Arab clients in mental health settings. Health Soc Work. 2000;25:9-22.

18. El-Islam M. Culture in the clinical practice of psychiatry. J Psychiatry. 2004; 15(1):8-16.

19. Arksey H, O'Malley L. Scoping studies: towards a methodological framework. Int J Soc Res Methodol. 2005;8:19-32.

20. Mays N, Pope C, Popay J. Systematically reviewing qualitative and quantitative evidence to inform management and policy-making in the health field. J Health Serv Res Policy. 2005:10:6-20.

21. Akers J. Systematic reviews: CRD's guidance for undertaking reviews in health care. York: CRD, University of York; 2009.

22. Anderson S, Allen P, Peckham S, Goodwin N. Asking the right questions: Scoping studies in the commissioning of research on the organisation and delivery of health services. Health Res Policy Syst. 2008;6:7.

23. Davis K, Drey N, Gould D. What are scoping studies? A review of the nursing literature. Int J Nurs Stud. 2009;46:1386-400.

24. Han CS, Oliffe JL, Ogrodniczuk JS. Suicide among East Asians in North America: A scoping review. J Mental Health. 2013;22:361-71.

25. Jenkins E, Goldner EM. Approaches to understanding and addressing treatment-resistant depression: a scoping review. Depress Res Treat. 2012; 2012:469680.

26. Ross F, Smith E, Mackenzie A, Masterson A. Identifying research priorities in nursing and midwifery service delivery and organisation: a scoping study. Int J Nurs Stud. 2004:41:547-58.

27. Saunders SL, Nedelec B. What work means to people with work disability: a scoping review. J Occup Rehabil. 2014;24(1):100-10.

28. World Health Organization. The Global Burden of Disease: 2004 Update. Geneva: WHO; 2008

29. Ghuloum S, Bener A, Abou-Saleh MT. Prevalence of mental disorders in adult population attending primary health care setting in Qatari population. J Pak Med Assoc. 2011;61:216-21.
30. Ritchie J, Lewis J. Qualitative research practice: a guide for social science students and researchers. Thousand Oaks: Sage Publications; 2003.

31. Bener A, Ghuloum S. Gender difference on patients' satisfaction and expectation towards mental health care. Niger J Clin Pract. 2013;16:285-91.

32. Amin Y, Hamdi E. Gender differences in psychiatric admissions: The influence of culture and social structure. Arab J Psychiatry. 1995;6(1):66-81.

33. Salem MO, Saleh B, Yousef S, Sabri S. Help-seeking behaviour of patients attending the psychiatric service in a sample of United Arab Emirates population. Int J Soc Psychiatry. 2009;55:141-8

34. Al-Solaim L, Loewenthal K. Religion and obsessive-compulsive disorder (OCD) among young Muslim women in Saudi Arabia. Ment Health Relig Cult. 2011;14:169-82.

35. Abalkhail B. Social status, health status and therapy response in heroin addicts. East Mediterr Health J. 2001;7:465-72.

36. Al-Ansari EA, Negrete JC. Screening for alcoholism among alcohol users in a traditional Arab Muslim society. Acta Psychiatr Scand. 1990;81:284-8.

37. Al-Kandari F, Yacoub K, Omu F. Effect of drug addiction on the biopsychosocial aspects of persons with addiction in Kuwait: nursing implications. J Addictions Nursing. 2007;18:31-40.

38. Bilal AM, Kristof J, El-Islam MF. A cross-cultural application of a drinking behaviour questionnaire. Addict Behav. 1987;12:95-101.

39. Daradkeh F, Moselhy HF. Death anxiety (Thanatophobia) among drug dependents in an Arabic psychiatric hospital. Am J Drug Alcohol Abuse. 2011;37:184-8.

40. Iqbal N. Recoverable hearing loss with amphetamines and other drugs. J Psychoact Drugs. 2002;36:285-8.

41. Qureshi NA. Trihexyphenidyl (artane) abuse among Saudi psychiatric patients. Ann Saudi Med. 1992;12:391-4.

42. Zaidan ZAJ, Dorvlo ASS, Viernes N, Al-Suleimani A, Al-Adawi S. Hazardous and harmful alcohol consumption among non-psychotic psychiatric clinic attendees in Oman. Int J Ment Health Addict. 2006;5:3-15.

43. El-Islam MF, Moussa MA, Malasi TH, Suleiman MA, Mirza IA. Assessment of depression in Kuwait by principal component analysis. J Affect Disord. 1988; 14:109-14.

44. Hamdi E, Amin Y, Abou-Saleh MT. Problems in validating endogenous depression in the Arab culture by contemporary diagnostic criteria. J Affect Disord. 1997:44:131-43.

45. Suleiman RA, Khashaba AS, Al-Haddad M. Depressive symptoms among HIV positive drug users in Bahrain. Arab J Psychiatry. 2002;13(1):31-5.

46. Al-Habeeb AA, Sherra KS, Al-Sharqi AM, Qureshi NA. Assessment of suicidal and self-injurious behaviours among patients with depression. East Mediterr Health J. 2013;19:248-54.

47. Suleiman MA, Nashef AA, Moussa MAA, El-Islam MF. Psychosocial profile of the parasuicidal patient in Kuwait. Int J Soc Psychiatry. 1986;32:16-22.

48. El-Islam MF. Cultural aspects of morbid fears in Qatari women. Soc Psychiatry Psychiatric Epidemiol. 1994;29:137-40.

49. Mahgoub OM, Abdel-Hafeiz HB. Pattern of obsessive-compulsive disorder in eastern Saudi Arabia. Br J Psychiatry. 1991;158:840-2.

50. Shooka A, Al-Haddad MK, Raees A. OCD in Bahrain: a phenomenological profile. Int J Soc Psychiatry. 1998;44:147-52.

51. Al-Ansari EA, Emara MM, Mirza IA, El-Islam MF. Schizophrenia in ICD-10: a field trial of suggested diagnostic guidelines. Compr Psychiatry. 1989;30: 416-9.

52. Kent G, Wahass S. The content and characteristics of auditory hallucinations in Saudi Arabia and the UK: a cross-cultural comparison. Acta Psychiatr Scand. 1996:94:433-7.

53. Zahid MA, Ohaeri JU. Schizophrenia psychopathology in a Kuwaiti Arab sample. Psychopathology. 2010;43:345-56.

54. Zarrouk ET. The frequency of visual hallucinations in schizophrenic patients in Saudi Arabia. Br J Psychiatry. 1975;127:553-5.

55. Zarrouk ET. The usefulness of first-rank symptoms in the diagnosis of schizophrenia in a Saudi Arabian population. Br J Psychiatry. 1978;132:571-3.

56. Al-Habeeb T, Qureshi N. Smoking among male psychiatric outpatients in Saudi Arabia. Ann Saudi Med. 2000:20:218-23.

57. Chaleby K. Women of polygamous marriages in an inpatient psychiatric service in Kuwait. J Nerv Ment Dis. 1985:173:56-8.

58. Al-Faraj A, Al-Ansari A. What family caregivers of schizophrenic and nonpsychotic in-patients in Bahrain believe about mental illness. Arab J Psychiatry. 2002;13(1):26-30.

59. Al Sughayir MA. Dissociative experience in psychiatric out patients who have possession belief. Arab J Psychiatry. 2005;16(2):98-106. 
60. Daradkeh TK, Karim L. Predictors of employment status of treated patients with DSM-III-R diagnosis. Can logistic regression model find a solution? Int J Soc Psychiatry. 1994:40:141-9.

61. Al-Subaie A. Traditional healing experiences in patients attending a university outpatient clinic. Arab J Psychiatry. 1994;5(2):84-91.

62. Qureshi NA, Al-Amri AH, Abdelgadir MH, El-Haraka EA. Traditional cautery among psychiatric patients in Saudi Arabia. Transcultural Psychiatry. 1998;35:75-83.

63. El-Islam MF. Rehabilitation of schizophrenics by the extended family. Acta Psychiatr Scand. 1982;65:112-9.

64. Bilal AM, Kristof J, Shaltout A, El-Islam MF. Treatment of alcoholism in Kuwait: a prospective follow-up study. Drug Alcohol Depend. 1987;19:131-44.

65. Al-Nahedh N. Relapse among substance-abuse patients in Riyadh, Saudi Arabia. East Mediterr Health J. 1999:5:241-6.

66. Demerdash AM, Mizaal H, El Farouki S, El Mossalem H. Some behavioural and psychological aspects of alcohol and drug dependence in Kuwait Psychiatric Hospital. Acta Psychiatr Scand. 1981;63:173-85.

67. Schoonover J, Lipkin S, Javid M, Rosen A, Solanki M, Shah S, et al. Perceptions of traditional healing for mental illness in rural Gujarat. Ann Glob Health. 2014:80:96-102.

68. Ae-Ngibise K, Cooper S, Adiibokah E, Akpalu B, Lund C, Doku V, et al. "Whether you like it or not people with mental problems are going to go to them": a qualitative exploration into the widespread use of traditional and faith healers in the provision of mental health care in Ghana. Int Rev Psychiatry. 2010;22:558-67.

69. Patel V S Simunyu E, Gwanzura F. The pathways to primary mental health care in high-density suburbs in Harare, Zimbabwe. Soc Psychiatry Psychiatr Epidemiol. 1997;32:97-103.

70. Lim A, Hoek HW, Blom JD. The attribution of psychotic symptoms to jinn in Islamic patients. Transcult Psychiatry. 2015;52:18-32.

71. Hanely J, Brown A. Cultural variations in interpretation of postnatal illness: jinn possession amongst Muslim communities. Community Ment Health J. 2013:50:348-53

72. Kirmayer LJ. Cultural variations in the clinical presentation of depression and anxiety: implications for diagnosis and treatment. J Clin Psychiatry. 2001;62:22-30.

73. Slade M. Personal Recovery and Mental Illness: A Guide for Mental Health Professionals. 1st ed. Cambridge. New York: Cambridge University Press; 2009

74. Uher R, Rutter M. Basing psychiatric classification on scientific foundation: problems and prospects. Int Rev Psychiatry. 2012;24:591-605.

75. Department of Health and Human Services. Mental health: Culture, race, and ethnicity. Supplement to mental health: a report of the Surgeon General. Washington: Government Printing Office, U.S. Public Health Service; 2001

76. Rosendal M, Jarbøl DE, Pedersen AF, Andersen RS. Multiple perspectives on symptom interpretation in primary care research. BMC Fam Pract. 2013;14:167.

77. Kleinman A. Rethinking Psychiatry: From Cultural Category to Personal Experience. 1st ed. New York. London: Free Press; 1991.

78. Afifi MM. Mental health publications from the Arab world cited in PubMed, 1987-2002. East Mediterr Health J. 2005;11:319-28.

79. Osman OT, Afifi M. Troubled minds in the Gulf: mental health research in the United Arab Emirates (1989-2008). Asia Pac J Public Health. 2010;22:48S-53.

80. Derbas A, Al-Haddad M. Factors associated with immediate relapse among Bahraini heroin abusers. East Mediterr Health J. 2001;7:473-80.

81. Al-Kandari F, Yacoub K, Omu F. Initiation factors for substance abuse. J Adv Nurs. 2001;34:78-85.

82. Al-Saffar N, Abdulkareem A, Abdulhakeem A, Salah A-Q, Heba M. Depressed patients' preferences for education about medications by pharmacists in Kuwait. Patient Educ Couns. 2008:72:94-101.

83. Bilal AM. Correlates of addiction-related problems in Kuwait: a cross-cultural view. Acta Psychiatr Scand. 1988;78:414-6.

84. El-Islam MF, Malasi TA, Suleiman MA, Mirza IA. The correlates of hypochondriasis in depressed patients. Int J Psychiatry Med. 1988;18:253-61.

85. Suleiman MA, Malasi TH, Mirza IA, El-Islam MF. Some characteristics of the psychiatric population attending a primary care centre in Kuwait. Acta Psychiatr Scand. 1989;79:199-204

86. Zahid M, Ohaeri J, Elshazly A, Basiouny M, Hamoda H, Varghese R. Correlates of quality of life in an Arab schizophrenia sample. Soc Psychiatry Psychiatr Epidemiol. 2010;45:875-87.

87. Zahid M, Ohaeri JU. Relationship of family caregiver burden with quality of care and psychopathology in a sample of Arab subjects with schizophrenia. BMC Psychiatry. 2010;10:71.
88. Zahid M, Ohaeri JU. Clinical and psychosocial factors associated with needs for care: an Arab experience with a sample of treated community-dwelling persons with schizophrenia. Soc Psychiatry Psychiatr Epidemiol. 2013;48:313-23.

89. Zahid M, Ohaeri JU, Al-Zayed AA. Factors associated with hospital service satisfaction in a sample of Arab subjects with schizophrenia. BMC Health Serv Res. 2010;10:294.

90. Bener A, Dafeeah EE, Salem MO. A study of reasons of non-compliance of psychiatric treatment and patients' attitudes towards illness and treatment in Qatar. Issues Ment Health Nurs. 2013:34(4):273-80.

91. Bener A, Al-Kazaz M, Ftouni D, Al-Harthy M, Dafeeah EE. Diagnostic overlap of depressive, anxiety, stress and somatoform disorders in primary care. Asia Pac Psychiatry. 2013;5:E29-38.

92. Chaleby KS, Raslan A. Delineation of social phobia in Saudia Arabians. Soc Psychiatry Psychiatr Epidemiol. 1990;25(6):324-7.

93. Chaleby KS. Psychosocial stresses and psychiatric disorders in an outpatient population in Saudi Arabia. Acta Psychiatr Scand. 1986;73:147-51.

94. Chaleby KS. Perceived parental attitude in social phobia patients. Ara. 1990;1.

95. Shahin SH, Daly EB. Knowledge, attitudes and beliefs about psychotropic medication among Saudi hospitalized psychiatric patients. Int J Nurs Stud. 1999:36:51-5.

96. Al-Sughayir MA. In-patient treatment for resistant obsessive-compulsive disorder. Saudi Med J. 2000:21:193-5.

97. Al-Showkan A, Curtis J, White Y. Self-reported quality of life for people with schizophrenia in a psychiatric outpatient department in Saudi Arabia. Arab J Psychiatry. 2013;24:93-101.

98. Chaleby K. Traditional Arabian marriages and mental health in a group of outpatient Saudis. Acta Psychiatr Scand. 1988;77:139-42.

99. Daradkeh TK, Al HT. Quality of life of patients with schizophrenia 2. East Mediterr Health J. 2005;11:898-904.

100. Al-Zarrad F, Abu-Mugaiseeb AM. Abu-Dhabi psychiatric day hospital efficacy and acceptability of its health service (a prospective study). Arab J Psychiatry. 2002;13(1):64-9.

\section{Submit your next manuscript to BioMed Central and we will help you at every step:}

- We accept pre-submission inquiries

- Our selector tool helps you to find the most relevant journal

- We provide round the clock customer support

- Convenient online submission

- Thorough peer review

- Inclusion in PubMed and all major indexing services

- Maximum visibility for your research

Submit your manuscript at www.biomedcentral.com/submit 